\title{
Wer erhält einen Ernährerlohn? Befunde nach Region und Geschlecht
}

\author{
Angesichts der in jüngerer Zeit kontrovers diskutierten Befunde einer „schrumpfenden \\ Mittelschicht" und zunehmender Lohnungleichheit ist offen, für wen der Lohn noch hin- \\ reichend ist, um die Existenz einer Familie zu sichern. Und in der Tat ist dies für einen \\ Teil der Gruppe berufsfachlich qualifizierter Vollzeitbeschäftigter infrage gestellt. Offen- \\ sichtlich gibt es relevante Lohnunterschiede zwischen einer Erzieherin und einem Metall- \\ facharbeiter, aber auch zwischen West- und Ostdeutschland und in Abhängigkeit von der \\ Interessenvertretung der Arbeitnehmer. ${ }^{\bullet}$
}

TIM SCHRÖDER, ANDREA SCHÄFER

\section{Einleitung}

In Deutschland sind die Einkommen aus unselbstständiger Erwerbsarbeit die bedeutendste Einkommensquelle privater Haushalte (Statistisches Bundesamt 2012). Das Erwerbssystem ist somit „der zentrale Ort der Verteilung von Lebenschancen und sozialer Sicherheit" (Lohmann/Andreß 2011, S. 178). Seit einiger Zeit jedoch wird ein zunehmender Bedeutungsverlust des sich in den 1950er Jahren flächendeckend institutionalisierenden, sogenannten „Normalarbeitsverhältnisses“, d.h. von abhängig Beschäftigten in unbefristeter, sozialversicherungspflichtiger Vollzeitbeschäftigung, diagnostiziert (Dietz/Walwei 2006). ${ }^{2}$ Zudem haben sich seit Mitte der 1990er Jahre die Löhne zugunsten eines wachsenden Niedriglohnsektors weiter auseinanderentwickelt (Dustmann et al. 2009). Darüber hinaus zeichnet sich seit den 2000er Jahren ein Trend stagnierender, zeitweilig sogar sinkender Reallöhne ab (Brenke/Grabka 2011). Aktuelle Studien weisen Reallohnverluste und zunehmende Niedriglöhne auch für Normalarbeitnehmer aus (ebd.). Damit ist der Kern des bundesdeutschen Erwerbssystems - Arbeitnehmer in Normalarbeitsverhältnissen - nicht nur von Beschäftigungs-, sondern zunehmend auch von Lohnunsicherheiten betroffen.

Im Gegensatz zur aktuellen Situation war das ,goldene Zeitalter" der ersten Jahrzehnte des bundesrepublikanischen Wohlfahrtsstaates noch durch dauerhafte Beschäftigungssicherheit und Reallohnsteigerungen gekennzeichnet.
Zugleich war bei den Sozialpartnern wie auch in Politik und Gesellschaft die Vorstellung vorherrschend, dass der Lohn, der in einem Normalarbeitsverhältnis erzielt wurde, den Bedarf einer ganzen Familie durchschnittlicher Größe, einer sogenannten Normalfamilie, decken müsste (siehe Gottschall/Schröder in diesem Heft). Ein solcher „Ernährerlohn" wurde typischerweise von einem berufsfachlich qualifizierten, männlichen Facharbeiter im Industriesektor mit starker Tarifbindung erwirtschaftet und hatte Vorbildcharakter für die Lohnfindung in anderen Bereichen. Unterstützt wurde die Vorstellung des Ernährerlohns durch sozialpolitische Regulierungen, die, insbesondere im

\footnotetext{
Wir danken den Koordinatorinnen dieses Schwerpunktheftes, Irene Dingeldey und Karin Gottschall, sowie Henning Lohmann und Markus Grabka für wertvolle Hinweise und Anregungen. Der Beitrag entstand im Rahmen des von der Hans-Böckler-Stiftung finanzierten Projektes „Was kommt nach dem Familienlohn (Projektnr. 2010-375-4).

(2) Die nachfolgende Untersuchung lehnt sich an die engere Definition von Normalarbeitsverhältnissen nach Mückenberger (1989) an, die nicht nur auf die volle Verfügbarkeit der Beschäftigten durch Vollzeiterwerbstätigkeit, sondern auch auf das Vorhandensein einer berufsfachlichen Qualifikation abhebt (vgl. Abschnitt 3). Wenn wir im Folgenden den Begriff Normalarbeitnehmer wie auch andere männlichen Formen verwenden, wird damit auf Frauen und Männer verwiesen.
} 
Rahmen der Ehe mit abgeleiteten sozialen Sicherungsansprüchen und Steuererleichterungen, ein traditionelles Ernährermodell mit nicht erwerbstätiger Hausfrau und Mutter begünstigten (Hinrichs 1996).

Angesichts veränderter Familien- und Geschlechterleitbilder, der Pluralisierung von Lebensformen sowie Umbrüchen in den Standards geschlechtsspezifischer Arbeitsteilung verliert das männliche Ernährermodell zugunsten anderer Familienerwerbsmuster an Bedeutung (Peukert 2008; Mayer-Ahuja et al. 2012). Mit zum Teil sinkenden Löhnen und steigender Frauenerwerbstätigkeit, vor allem im Dienstleistungssektor, rückt die Frage in den Fokus, ob und inwieweit Ernährerlöhne über die industriellen Kernsektoren hinaus auch auf andere Branchen übertragbar sind und inwieweit Frauen im Vergleich zu Männern Ernährerlöhne erzielen. Dies ist auch angesichts der unterschiedlichen Geschichte beider deutscher Staaten relevant; denn anders als in der alten Bundesrepublik Deutschland (BRD) war in der Deutschen Demokratischen Republik (DDR), bei niedrigem Lohnniveau ein Zweiverdienermodell mit doppelter Vollzeitbeschäftigung vorherrschend - ein Familienerwerbsmuster, das auch heute noch in Ostdeutschland dominiert (Klenner et al. 2012).

$\mathrm{Zu}$ dieser Frage liegen bisher nur wenige Erkenntnisse vor. Einige Studien untersuchen die familiale Existenzsicherung durch Individuallöhne. Strengmann-Kuhn (2003, S. 112ff.) etwa ermittelt den Anteil an armen Erwerbstätigen, bei denen der Lohn die auf Basis des mittleren verfügbaren Einkommens einer Normalfamilie errechnete Armutsgrenze nicht überschreitet. Andreß und Seeck (2007) wiederum fokussieren auf Normalarbeitnehmer, verwenden jedoch individuelle (nach Haushaltsgröße gewichtete) Armutsgrenzen. Die vorliegende Analyse schließt hier mit dem Fokus auf Normalarbeitnehmer an, konzentriert sich jedoch auf die Verbreitung und Bedingungen von Ernährerlöhnen, d. h. eine am realen oder potenziellen Ernährerstatus orientierte Existenzsicherung qua individuellem, männlichem wie auch weiblichem Lohneinkommen. Besondere Berücksichtigung finden, neben der Ost-Westdifferenz, die geschlechtsspezifische Arbeitsmarktsegregation und institutionelle Interessenvertretung. Im Einzelnen wird untersucht, inwiefern gegenwärtig in Ost- und Westdeutschland von Frauen und Männern in Normalarbeitsverhältnissen Ernährerlöhne erzielt werden, wie sich der Anteil von Normalarbeitnehmern mit Ernährerlöhnen im Zeitverlauf entwickelt hat und unter welchen branchenspezifischen Bedingungen das Erwirtschaften eines Ernährerlohns gelingt.

Der Beitrag beginnt mit einer Erörterung der geschlechtsspezifischen Lohndifferenzen in West- und Ostdeutschland, des Zusammenhangs mit geschlechtsspezifischer Arbeitsmarktsegregation und institutioneller Interessenvertretung sowie der Ableitung von Hypothesen zum Ernährerlohn (Abschnitt 2). Im Anschluss an die Dokumentation des verwendeten Datensatzes sowie der methodischen Anlage der Untersuchung (3) erfolgt eine Deskriptivanalyse der Entwicklung des Ernährerlohns von Normalarbeitnehmern in
West- und Ostdeutschland von Mitte der 1990er Jahre bis 2008 (4). Schließlich werden die regionalen und geschlechtsspezifischen Differenzen im Ernährerlohn anhand einer Logit-Regression für das Jahr 2008 auf branchentypische und betriebliche geschlechtsspezifische Segregation einerseits sowie die Tarifbindung andererseits zurückgeführt (5). Zum Schluss werden die wichtigsten Ergebnisse und deren mögliche Implikationen diskutiert (6).

\section{Lohndifferenzen nach Region und Geschlecht und ihre Ursachen}

Die Frage nach der geschlechtsspezifischen Entwicklung des Ernährerlohns von Normalarbeitnehmern in West- und Ostdeutschland hat dem Umstand Rechnung zu tragen, dass es sich um zwei ehemals unabhängige Staaten handelt. Hier haben sich unterschiedliche Formen der Lohnfindung und "Geschlechterregimes“ (Lewis/Ostner 1994) etabliert, die - trotz wechselseitiger Annäherungsprozesse seit Beginn der 1990er Jahre - noch heute wirksam sind.

Die alte $B R D$ kann zunächst als eine institutionalisierte „Hochlohn-Ökonomie“ mit einem vergleichsweise hohen, in den ersten Jahrzehnten real stark ansteigenden Lohnniveau bezeichnet werden (Streeck 1995). Komplementär hierzu war das männliche Ernährermodell weit verbreitet. Frauenerwerbstätigkeit galt lange Zeit als Notwendigkeit in Familien, denen ein Erwerbseinkommen nicht ausreichte (Gottschall/Schröder in diesem Heft). Das (immer schon) niedrige Lohnniveau der Frauen wurde nicht zuletzt durch den geringen Bedarf bei Frauen ohne Familie bzw. den $\mathrm{Zu}$ verdienstcharakter der Erwerbstätigkeit von Frauen mit Familie legitimiert (ebd.). Noch zu Beginn der 1990er Jahre herrschte in westdeutschen Paarhaushalten das klassische Ernährermodell des männlichen Alleinverdieners in Vollzeitbeschäftigung vor (Trappe/Sørensen 2006). Die Lohndifferenz zwischen Männern und Frauen, also die geschlechtsspezifische Lohnlücke (gender pay gap), war auch im internationalen Vergleich groß (Szydlik 1993). Seither ist die Lohnentwicklung auf dem westdeutschen Arbeitsmarkt durch Stagnation und zeitweilig sogar Reallohnverluste gekennzeichnet (Roos 2006). Daneben kommt es zu einer starken Zunahme der Teilzeitbeschäftigung von Frauen. Infolgedessen verliert das klassische männliche Ernährermodell an Bedeutung (vgl. Berninger/Dingeldey in diesem Heft). Die geschlechtsspezifische Lohnlücke bleibt dennoch bestehen (Finke 2011).

Das Wirtschaftssystem der DDR hingegen war durch ein geringes Lohnniveau wie auch eine geringere Lohnungleichheit gekennzeichnet (Szydlik 1993). Die chronische Arbeitskräfteknappheit führte in Verbindung mit den sozialistischen Ideen der Gleichstellung schon bis zu Beginn der 1970er Jahre zu einer vollen Etablierung des Zweiverdienermodells mit Vollzeitbeschäftigung für beide Partner 
bei staatlicher Kinderbetreuung (Trappe/Sørensen 2006). Dennoch bestand bis zum Ende der DDR eine geschlechtsspezifische Lohnlücke - zwar geringer als in der BRD, angesichts der allgemein niedrigen Lohnungleichheit jedoch beträchtlich (Winkler 1990). Hinzu kommt, dass auch in der DDR die traditionelle geschlechtsspezifische Arbeitsteilung im Haushalt erhalten blieb und Frauen somit eine Doppelbelastung zu tragen hatten (ebd.). Insofern trug das Zweiverdienermodell deutlich geschlechtsspezifische Züge.

Mit der Wirtschafts- und Währungsunion setzte ein radikaler Transformationsprozess ein, der sich erst bis Mitte der 1990er Jahre konsolidierte (Falk 2005). Die Erwerbstätigen in Ostdeutschland konnten in den ersten Jahren noch große Reallohngewinne verbuchen, seither jedoch stagniert die Annäherung an Westdeutschland (Roos 2006). Hinzu kommen ein starker Anstieg der Erwerbslosigkeit, ein Rückgang der Vollzeitbeschäftigung und ein damit einhergehender Bedeutungsverlust des klassischen Zweiverdienermodells, das aber nach wie vor dominiert (Klenner et al. 2012). Bei den Erwerbstätigen steigt vor allem der Anteil von „Familienernährerinnen“, d. h. weiblicher Alleinverdiener inklusive Alleinerziehender, die mittlerweile deutlich häufiger sind als in Westdeutschland (ebd.). Bemerkenswerterweise reduziert sich in Ostdeutschland seit 1990 die geschlechtsspezifische Lohnlücke und liegt deutlich unter Westniveau (Hunt 2002).

Vor diesem Hintergrund ergeben sich folgende Hypothesen zu regionalen und geschlechtsspezifischen Differenzen im Ernährerlohn: Aufgrund eines niedrigen Lohnniveaus in Kombination mit einem dominanten Zweiverdienermodell ist zu erwarten, dass in Ostdeutschland seltener Ernährerlöhne erzielt werden als in Westdeutschland (Hypothese 1a). $\mathrm{Zu}$ erwarten ist auch, dass Frauen insgesamt seltener einen Ernährerlohn erwirtschaften als Männer. Aufgrund der noch heute im Erwerbssystem nachwirkenden, starken Tradition des Ernährermodells sollte diese geschlechtsspezifische Differenz im Bereich der Ernährerlöhne in Westdeutschland besonders groß ausfallen. Wenngleich sich die geschlechtsspezifische Lohnlücke in Ostdeutschland aktuell schließt, so ist im Bereich der Ernährerlöhne dennoch mit einer, wenn auch geringeren Benachteiligung der weiblichen Normalarbeitnehmer zu rechnen (Hypothese $1 b$ ). ${ }^{8}$

Die Lohnstruktur in Deutschland wird in starkem Maße einerseits durch die Beschäftigungsstruktur in den Branchen und Betrieben und andererseits durch die institutionelle Lohnsetzung kollektiver Akteure beeinflusst. Fragt man daher nach den hinter den regionalen Geschlechterdifferenzen liegenden Determinanten der Verbreitung von Ernährerlöhnen, so ist erstens die geschlechtsspezifische Arbeitsmarktsegregation zu nennen, d. h. die strukturelle Konzentration von Männern und Frauen in spezifischen Branchen und Betrieben. Historisch war die geschlechtsspezifische Segregation in der alten BRD im internationalen Vergleich hoch und wurde für ein gewisses Ausmaß an Lohnungleichheit verantwortlich gemacht (Falk 2005). Kern der Wirtschaft war die Industrie, in der vorwiegend
Männer tätig waren. Die Tätigkeiten dort galten als äußerst „produktiv“ und wurden entsprechend hoch entlohnt. Die im Industriesektor beschäftigten Frauen waren indes vorwiegend in Branchen tätig, die als eher unproduktiv galten - nicht zuletzt deshalb, weil dort, wie in der Textilbranche, ehemals in unbezahlter Hausarbeit verrichtete Tätigkeiten ausgeübt wurden (Beck-Gernsheim 1981). Doch auch die wenigen, in den männlich geprägten industriellen Kernbranchen beschäftigten Frauen erreichten nur selten (männliche) Facharbeiterlöhne (Lappe 1981). Die Frauenerwerbstätigkeit insgesamt konzentrierte sich vorwiegend auf personenbezogene bzw. soziale Dienstleistungen wie Pflege- und Erziehungstätigkeiten im Gesundheits- und Sozialwesen. Es handelte sich hierbei vor allem um ehemals aus dem Haushaltskontext ausgegliederte „unproduktive“ Dienstleistungen mit entsprechend niedriger Entlohnung. Noch bis in die 2000er Jahre hinein blieb die geschlechtsspezifische Segregation in Westdeutschland persistent (Falk 2005; Heinze 2009). Doch auch und gerade in der DDR bestand eine, selbst im Vergleich zur BRD starke geschlechtsspezifische Arbeitsmarktsegregation (Trappe/ Rosenfeld 2001). Die modernen Grundstrukturen der industriell-fordistischen Produktion und die damit einhergehende „Facharbeitertradition“ blieben erhalten und wurden weiter gepflegt (Busch/Land 2012). Dabei implizierte die Planwirtschaft eine staatlich organisierte und betrieblich umgesetzte Beschäftigungslenkung sowie Lohnsetzung durch die (überwiegend männlichen) Führungskader, welche die Frauen, trotz offizieller Gleichstellungspolitik, an Branchen mit niedrigen Löhnen verwies (Roloff 1991). Nach der Wende nahm die geschlechtsspezifische Lohnungleichheit bis Ende der 1990er Jahre stetig ab, während die geschlechtsspezifische Arbeitsmarktsegregation zunächst zu und dann abnahm (Trappe/Rosenfeld 2001). Die Zunahme der Segregation wurde auf den Arbeitsplatzverlust niedrig qualifizierter und entlohnter Frauen in den industriellen Kernsektoren zurückgeführt (Hunt 2002); die Abnahme war hingegen durch die steigende Männerbeschäftigung in den DDR-typischen Frauenberufen des Dienstleistungssektors (etwa Banken und Versicherungen) bedingt (Schlegel 1995). Auf betrieblicher Ebene hingegen zeigte sich eindeutig ein negativer Effekt von Betrieben mit hohem Frauenanteil auf das Lohnniveau für West- und Ostdeutschland (Heinze 2009). Im Anschluss an diese Überlegungen wird für beide Regionen erwartet, dass in Branchen und Betrieben mit hohem Frauenanteil seltener Ernährerlöhne gezahlt werden (Hypothese 2a). Gemäß der Dominanz des männlichen Ernährermodells sollten Frauen in Westdeutschland, unabhängig davon ob sie in Frauen- oder Männerbranchen bzw. -betrieben tätig sind,

3 Mit dem Buchstaben „a“ gekennzeichnete Hypothesen spezifizieren im Folgenden regionale Differenzen, Hypothesen mit dem Buchstaben "b" einen geschlechtsspezifischen Unterschied, der jeweils regional differenziert wird. 
gegenüber Männern seltener Ernährerlöhne erzielen. In Ostdeutschland hingegen sollten Männer ebenso wie Frauen in Frauenbranchen und -betrieben seltener einen $\mathrm{Er}$ nährerlohn erhalten als in Männerbranchen/-betrieben (Hypothese 2b).

Neben der geschlechtsspezifischen Arbeitsmarktsegregation spielt zweitens die Lohnsetzung der institutionellen Interessenvertretung, insbesondere die Tarifbindung, eine Rolle für den Ernährerlohn. Die industriellen Kernsektoren in Westdeutschland sind institutionell durch starke Gewerkschaften und kollektive Lohnverhandlungen gekennzeichnet. Die in „Lohnführerschaft" wegweisend auch für andere Wirtschaftszweige ausgehandelten Facharbeiterlöhne wurden in den 1950er Jahren wie selbstverständlich mit einem Ernährerlohn gleichgesetzt (siehe Gottschall und Schröder in diesem Heft). Die Reproduktion des männlichen Ernährermodells im Erwerbssystem führt dabei dazu, dass Frauen seltener in Betrieben tätig sind, die durch branchenweit ausgehandelte Tarifverträge geschützt sind (Antonczyk et al. 2011). Andererseits können sie dort, wo ein solcher Schutz besteht, besonders profitieren (ebd.). Im Gegensatz zur alten BRD wurden die „freien Gewerkschaften“ in der DDR staatlich inkorporiert, die Lohnsetzung folgte der staatspolitischen Programmatik von Bedarfssicherung bei geringer Ungleichheit (Busch/Land 2012). Dabei wurde zu Beginn der 1950er Jahre das bestehende, die Frauen benachteiligende Tarifsystem übernommen und seither kaum verändert (Roloff 1991). Die Geschlechterungleichheit in den Löhnen blieb somit erhalten. Mit der Wirtschafts- und Währungsunion galt das westdeutsche Modell der Tarifautonomie für beide Regionen gleichermaßen. Angesichts fehlender Gewerkschafts-, aber auch Arbeitgeberstrukturen nahmen die Gewerkschaften in Westdeutschland einen starken Einfluss auf die Tarifpolitik in Ostdeutschland, die vor allem eine Politik der Lohnangleichung war (Krueger/ Pischke 1995). Die Tarifbindung erreichte allerdings nie das westdeutsche Niveau. In beiden Landesteilen erodiert seit Mitte der 1990er Jahre das System institutioneller Interessenvertretung (Bonin 2005). Dies führt zum Teil zu Reallohnverlusten in Westdeutschland sowie einer Stagnation der Löhne in Ostdeutschland (Dustmann et al. 2009). Vor diesem Hintergrund ist zu vermuten, dass in Ost- wie Westdeutschland Normalarbeitnehmer in tarifgebundenen Betrieben eher Ernährerlöhne erzielen als in Betrieben ohne Tarifbindung (Hypothese 3a). Da Frauen von der lohnnivellierenden Wirkung der Tarifbindung profitieren, ist davon auszugehen, dass eine Erosion der Tarifbindung zu einer Zunahme der Geschlechterdifferenzen hinsichtlich der Löhne führt. Unklar ist dennoch, ob die Tarifbindung gleiche schützende Wirkungen für Frauen am oberen Ende der Lohnverteilung, bei den Ernährerlöhnen, wie im Bereich der Tariflöhne zeigt. Insgesamt lässt sich vermuten, dass im Falle von Ernährerlöhnen eher die Männer - vor allem in Westdeutschland - von der Tarifbindung profitieren (Hypothese $3 b$ ).

\section{Daten, Variablen und Methode}

Die Überprüfung der Hypothesen erfolgt mithilfe des Linked Employer-Employee Datensatzes des Instituts für Arbeitsmarkt und Berufsforschung (IAB) der Bundesagentur für Arbeit (kurz: LIAB). ${ }^{\circledR}$ Beim LIAB handelt es sich um repräsentative Daten für deutsche Betriebe, die mit Informationen über alle in diesen Betrieben sozialversicherungspflichtig Beschäftigten kombiniert werden. ${ }^{\ominus}$ Die vorliegende Stichprobe enthält nur erwachsene Personen im Alter von 25 bis 64 Jahren. Episoden parallelen Leistungsbezugs der Bundesagentur wie auch Beschäftigte in Altersteilzeit, Ausbildung, mithelfende Familienangehörige sowie unständig Beschäftigte werden ausgeschlossen. Aus den Jahresquerschnitten wird ein unbalancierter Paneldatensatz erstellt. Als Ausgangszeitpunkt wird das Jahr 1996 gewählt, an dem der ostdeutsche Transformationsprozess sich bereits konsolidiert hatte. Der Endzeitpunkt im Jahr 2008 liegt möglichst nah am aktuellen Rand, ohne die globale Finanzkrise und deren Auswirkungen auf die Löhne abzubilden.

Beschäftigte in Normalarbeitsverhältnissen werden anhand zweier Kenngrößen definiert: Erwerbsumfang und Qualifikation. Normalarbeitnehmer haben demnach erstens eine abhängige, sozialversicherungspflichtige Vollzeitbeschäftigung inne. Da der LIAB keine Informationen über die Befristung enthält, ist es nicht möglich, Normalarbeitnehmer zusätzlich anhand des Attributes „unbefristet“ des Arbeitsvertrages zu identifizieren. ${ }^{\circledR}$ Zweitens wird die Gruppe der Normalarbeitnehmer, um den Kern des Beschäftigungssystems zu erfassen, über eine mittlere, berufsfachliche Qualifikation bestimmt, d. h. eine berufliche Ausbildung oder einen Fachhochschulabschluss (vgl. Mückenberger 1989). Die Stichprobe der Normalarbeitnehmer umfasst (ungewichtet) für das Jahr 1996 insgesamt 1,2 Mio. und im Jahr 2008 insgesamt 0,8 Mio. Beschäftigte in Normalarbeitsverhältnissen.

Die abhängige Variable - der Ernährerlohn - wird gemäß den Erörterungen in Abschnitt 1 und 2 inhaltlich bestimmt als ein (Facharbeiter-)Lohn, der von einem männlichen, berufsfachlich qualifizierten Normalarbeitnehmer im männlich dominierten industriellen Kernsektor der Metall-

(4) Die Datengrundlage bildet das sogenannte "LIAB-Querschnittmodell 2" (siehe Jacobebbinghaus/Seth 2010). Der Datenzugang erfolgte über einen Gastaufenthalt am „FDZ im FDZ" des IAB im Statistischen Landesamt Bremen und anschließend mittels kontrollierter Datenfernverarbeitung.

(5) Der Datensatz ist nach Betriebsgröße, Branche und Bundesland geschichtet (zur Repräsentativität siehe Alda 2005).

6. Wie Dietz/Walwei (2006) zeigen, liegt der Anteil von befristeten Beschäftigten Mitte der 2000er Jahre bei unter $10 \%$. Zudem kann die Befristung auch eine „Brücke” in ein Normalarbeitsverhältnis sein. Schließlich sind vor allem jüngere Beschäftigte von unter 25 Jahren von Befristungen betroffen. 
oder Automobilbranche mindestens erreicht wird. Diese Branchen wiesen traditionell eine hohe Tarifbindung auf. In kollektiven Lohnverhandlungen wurden die Löhne für sogenannte Ecklohngruppen von Facharbeitern (mit Berufserfahrung) bestimmt und gemäß einem „Lohnschlüssel“ auf die anderen Gruppen verteilt (Beckord 1977). Zudem kam diesen Branchen die „Lohnführerschaft“ für eine Reihe anderer Branchen zu (ebd.). Empirisch ermittelt wird die Ernährerlohngrenze anhand des Medians ${ }^{0}$ des TagesBrutto-Reallohns der Männer in einer abhängigen, sozialversicherungspflichtigen Vollzeitbeschäftigung mit einer berufsfachlichen Ausbildung oder einem Fachhochschulabschluss, die in der Metall- oder Automobilbranche mit mindestens zweijähriger Betriebszugehörigkeit tätig sind. ${ }^{\circledR}$ Es liegt eine Ernährerlohngrenze für jedes Jahr vor. Bezogen auf einen Stundenlohn, bei einer angenommenen Wochenarbeitszeit von 39,5 Stunden, lag diese Grenze im Jahr 1996 bei $18 €$ und im Jahr 2008 bei $19 €$. Dies entspricht einem Monatslohn von $3.080 € \mathrm{im}$ Jahr 1996 und von $3.252 € \mathrm{im}$ Jahr 2008. Der Ernährerlohn liegt damit oberhalb der mittleren Löhne aller Normalarbeitnehmer. Diese betragen monatlich $2.707 €$ im Jahr 1996 und $2.737 €$ im Jahr 2008. Dabei wird von einer einheitlichen Ernährerlohngrenze für West- und Ostdeutschland ausgegangen, da Deutschland, was das Anspruchsniveau in Bezug auf Löhne betrifft, spätestens seit Mitte der 1990er Jahre als ein Gesamtarbeitsmarkt gelten kann (Hauser/Wagner 1996). Der Indikator Ernährerlohn erhält den Wert 1, wenn ein Normalarbeitnehmer einen Tages-Brutto-Reallohn erhält, der oberhalb der Ernährerlohngrenze liegt, ansonsten den Wert 0.

Für die Erklärung des Ernährerlohns wurden auf Basis der theoretischen Annahmen zwei Gruppen möglicher Prädiktoren berücksichtigt: Erklärungsbeiträge leisten erstens die geschlechtsspezifische Arbeitsmarktsegregation nach Branchen einerseits und Betrieben andererseits. Die Segregation der Branchen wird erfasst durch die Differenzierung von klassischen geschlechtstypischen Branchen: der Automobil- und Metallbranche (Frauenanteil: $11 \%$ ), des Gesundheits- und Sozialwesens (Frauenanteil: $75 \%$ ) und der Banken und Versicherungen (Frauenanteil: $45 \%$ ). ${ }^{\ominus}$ Mit der betrieblichen Segregation, gemessen über den Frauenanteil im Betrieb, wird zudem ein direkterer Bezug auf die Beschäftigungsstruktur im Betrieb genommen. Es lassen sich so etwa Betriebe mit einem hohen Frauenanteil innerhalb von männerdominierten Industriebranchen identifizieren. Es werden drei Gruppen gebildet: männerdominierte Betriebe mit einem Frauenanteil unter $33 \%$, integrierte Betriebe mit einem Frauenanteil zwischen 33 und $66 \%$ und frauendominierte Betriebe mit einem Frauenanteil von $66 \%$ und mehr (vgl. Falk 2005, S. 133). Zweitens fließen Informationen zu den institutionellen Strukturen der Interessenvertretung in die Untersuchung ein: So wird der Einfluss des Grades der Tarifbindung in die Analyse einbezogen. Unterschieden werden Betriebe mit Branchen- oder Firmentarifvertrag, Betriebe ohne Tarifvertrag, die sich jedoch am Branchentarif orientieren, sowie Betriebe ohne Tarifvertrag und ohne Orientierung am Branchentarif.

Zusätzlich werden theoretisch bedeutsame Kontrollvariablen aufgenommen, um der Heterogenität der Beschäftigten und der Betriebe Rechnung zu tragen. Zunächst bilden das Alter, die Berufsausbildung und die Betriebszugehörigkeitsdauer den Grad des Humankapitals ab. Die betriebliche Gelegenheitsstruktur wird mithilfe der Betriebsgröße, des Vorhandenseins eines Betriebsrates sowie des Anteils von Einstellungen, d. h. Anzahl der Personen, die neu eingestellt wurden, dividiert durch die Anzahl der sozialversicherungspflichtig Beschäftigten im Betrieb, erfasst. Mit dem Anteil von Einstellungen wird die Dynamik der Allokation von Arbeitskräften auf Arbeitsplätze abgebildet. ${ }^{\circledR}$

Mithilfe einer Logit-Regression (Long/Freese 2006) wird die Chance von Normalarbeitnehmern, einen Ernährerlohn zu erzielen, auf die beiden theoretisch bedeutsamen Erklärungsfaktoren, geschlechtsspezifische Arbeitsmarktsegregation (branchenspezifische und betriebliche) und Tarifbindung, zurückgeführt. So werden im Falle der geschlechtsspezifischen Arbeitsmarktsegregation etwa die Chancen von Normalarbeitnehmern, die in frauendominierten Branchen beschäftigt sind, ins Verhältnis zu den Chancen der Normalarbeitnehmer in männerdominierten Branchen gesetzt. Um der regionalen Spezifik des Ernährerlohns Rechnung zu tragen, werden
(7) Der Median teilt die nach Lohnhöhe sortierte, hier definierte Gruppe in zwei Hälften. Die eine Hälfte bekommt mehr, die andere weniger als den mittleren Lohn. Zu beachten ist, dass nach dieser Setzung somit die Hälfte der Beschäftigten in der Referenzgruppe, auf die sich das historische Konstrukt des Ernährerlohns bezieht, keinen Ernährerlohn erhält.

8 Grundlage der Variablen ist das Tages-Brutto-Entgelt, gemessen in Euro. Unplausible Fälle mit einem Entgelt unterhalb der Midijobgrenze von $800 €$ wurden dabei ausgeschlossen. Zudem wurden die nicht erfassten Entgelte oberhalb der Bemessungsgrenze zur Sozialversicherung geschätzt und imputiert (Gärtner/Rössler 2005). Um zeitliche sowie regionale Kaufkraftunterschiede zu korrigieren, ist schließlich sowohl die jährliche Preissteigerung als auch das Preisniveau zwischen Ost- und Westdeutschland (ohne Mieten) berücksichtigt. Es handelt sich demnach um RealEntgelte in westdeutschen Preisen von 2005.

(9 Eine Stichprobenbeschreibung sowie zusätzliche Tests auf Robustheit der Ergebnisse können bei den Autoren angefordert werden.

(10 Es ist zu beachten, dass die Lohnwirkung aller hier verwendeten substanziellen Erklärungsfaktoren (Segregation und Tarifbindung) wiederum durch weitere, im Rahmen dieser Studie nicht beobachtete Faktoren beeinflusst werden kann. So können im Falle der Segregation u. a. die Produktivität, im Falle derTarifbindung u. a. außertarifliche Zahlungen oder Leistungszulagen zusätzlich wirksam sein. 
Modelle getrennt für West- und Ostdeutschland dargestellt. Gemäß den Ausführungen in Abschnitt 2 sollten die beiden Erklärungsfaktoren geschlechtsspezifische Arbeitsmarktsegregation und Tarifbindung für Männer und Frauen unterschiedlich wirksam sein, d. h. Frauen sollten etwa in männerdominierten Branchen niedrigere Löhne erzielen als ihre Kollegen. Um dies zu prüfen, werden in einem weiteren Schritt die jeweiligen erklärenden Variablen mit der Variablen Geschlecht (hier Referenzkategorie Frau) multipliziert und zusätzlich in die Modelle mit aufgenommen.

\section{Die Entwicklung des Ernährerlohns nach Region und Geschlecht}

Knapp ein Viertel aller Normalarbeitnehmer der Stichprobe erwirtschaftete im Jahr 2008 einen Ernährerlohn (Abbildung 1).

Der Anteil ist seit 1996 um zwei Prozentpunkte gesunken. Trotz Angleichung der Anteile von Frauen und Männern in Ost- und Westdeutschland bleiben im gesamten Zeitraum große regionale wie auch geschlechtliche Differenzen im Erhalt des Ernährerlohns bestehen: Nicht nur Beschäftigte in Ostdeutschland, sondern auch Frauen erwirtschaften im Jahr 2008 weitaus seltener einen Ernährerlohn. Es sind damit in erster Linie die Männer in Westdeutschland, die einen Ernährerlohn erzielen - mit weitem Abstand zu den übrigen Normalarbeitnehmern. Allerdings

ABB.

\section{Entwicklung des Anteils von Normalarbeitnehmern mit Ernährerlohn* $1996-2008$}

$\begin{array}{lll}\text { Angaben in Prozent } & \text { - Westdeutschland Frauen } & - \text { Westdeutschland Männer } \\ \text {-- Deutschland gesamt } & - \text { Ostdeutschland Frauen } & - \text { Ostdeutschland Männer }\end{array}$

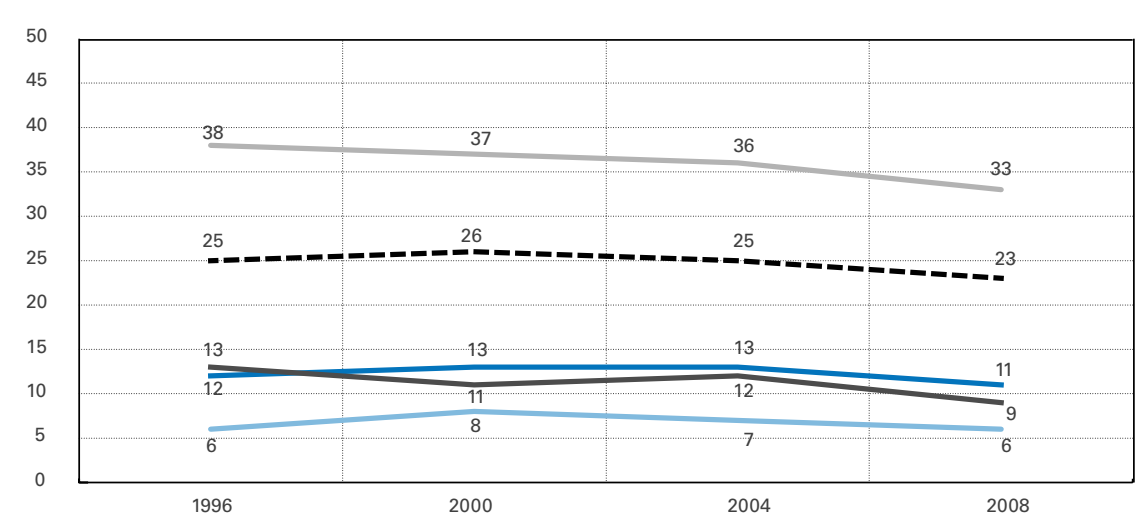

*nach Geschlecht und Region

Anmerkung: Normalarbeitnehmer (Personen in abhängiger, sozialversicherungspflichtiger Vollzeitbeschäftigung mit mittlerem Ausbildungsgrad); Ernährerlohn (Normalarbeitnehmer mit mindestens einem mittleren Tages-Brutto-Reallohn eines männlichen Normalarbeitnehmers in der Metall- oder Automobilbranche mit mindestens zweijähriger Betriebszugehörigkeit).

Quelle: LIAB Querschnittmodell (Version 2), 1996-2008, gewichtete Ergebnisse; sind bei den westdeutschen Männern in Normalarbeitsverhältnissen auch Veränderungen sichtbar: Der Anteil der Beschäftigten mit Ernährerlohn nimmt von 1996 bis 2008 um fünf Prozentpunkte von 38 auf $33 \%$ ab. Dies entspricht einem Rückgang um $13 \%$. Wenn auch in Westdeutschland in absoluten Zahlen mehr Männer von diesem Rückgang betroffen sind als in Ostdeutschland, fällt der Rückgang von 13 auf $9 \%$ (um vier Prozentpunkte) - dies entspricht einer Veränderung um $31 \%$ - dort, prozentual gesehen, stärker ins Gewicht. Es bestätigt sich, dass Beschäftigte in Ostdeutschland seltener einen Ernährerlohn erhalten als in Westdeutschland ( $\mathrm{Hla}$ ). Die geringsten Möglichkeiten, einen Ernährerlohn zu erwirtschaften, haben Frauen in Normalarbeitsverhältnissen in Ostdeutschland. Insgesamt zeigen die Ergebnisse eine klare Geschlechterrelation mit regionaler Prägung: Frauen können in beiden Regionen weitaus seltener einen Ernährerlohn erzielen als Männer. Dieser Befund findet seine stärkste Ausprägung bei den Normalarbeitnehmern in Westdeutschland; hier ist die Geschlechterdifferenz in den Ernährerlöhnen weitaus größer als in Ostdeutschland $(H 1 b)$.

\section{Ernährerlöhne, Tarifbindung und Segregation}

Auch nach Kontrolle weiterer individueller wie betrieblicher Merkmale anhand einer Logit-Regression für das Jahr 2008 bestätigt sich für beide Regionen das Bild geschlechtlicher wie regionaler Differenzen im Erhalt von Ernährerlöhnen (Tabelle 1).

Dabei spielt die strukturelle Benachteiligung durch die Positionierung innerhalb des Erwerbssystems anhand von Branchen eine wichtige Rolle. Eindeutig bestätigt sich, dass in beiden Regionen im frauendominierten Gesundheits- und Sozialwesen wesentlich geringere Chancen bestehen, einen Ernährerlohn zu erzielen, als in den industriellen Kernsektoren der Metall- und Automobilbranchen (H2a). Interessanterweise haben Beschäftigte im Banken- und Versicherungswesen in Ostdeutschland eine größere Chance, einen Ernährerlohn zu erwirtschaften, als Beschäftigte in der Metall- und Automobilindustrie. Es kann vermutet werden, dass die ostdeutsche Metall- und Automobilindustrie eine im Vergleich zu Westdeutschland geringere Produktivität und damit ein überproportional geringes Lohnniveau aufweist.

Mit dem betrieblichen Frauenanteil treten regionale Besonderheiten im Bezug von Ernährerlöhnen zutage: Für Beschäftigte in Westdeutschland spielt es eine große Rolle, ob der Betrieb, in dem sie arbeiten, vorrangig Frauen beschäftigt

(11) In einem Modell für Gesamtdeutschland bestätigt sich die Ost-West-Differenz (nicht dargestellt). Somit kann von strukturell differenten Regionen ausgegangen werden. 
TABELLE 1

\section{Determinanten des Ernährerlohns bei Normalarbeitnehmern 2008}

Logit-Regression, relative Chancen

\begin{tabular}{|c|c|c|}
\hline & Westdeutschland & Ostdeutschland \\
\hline Frau (Referenz: Mann) & $0,41 * * *$ & $0,46 * * *$ \\
\hline \multicolumn{3}{|l|}{ Segregation } \\
\hline \multicolumn{3}{|l|}{ Branchen (Referenz: Automobil- und Metallbranche) } \\
\hline Banken und Versicherungen & 1,54 & $2,11 * * *$ \\
\hline Gesundheits- und Sozialwesen & $0,18 * * *$ & $0,24 * * *$ \\
\hline \multicolumn{3}{|l|}{ Betrieblicher Frauenanteil (Ref.: Männerdominierter Betrieb) } \\
\hline Frauendominierter Betrieb & $0,34 * * *$ & 0,83 \\
\hline Integrierter Betrieb & $0,58 * *$ & 1,21 \\
\hline \multicolumn{3}{|l|}{ Institutionelle Interessenvertretung } \\
\hline \multicolumn{3}{|l|}{ Tarifbindung (Referenz: Branchen-Tarifvertrag) } \\
\hline Firmentarifvertrag & 1,11 & 0,93 \\
\hline Kein Tarifvertrag, Orientierung an Branchen-TV & $0,78 *$ & $0,57 * * *$ \\
\hline Kein Tarifvertrag, keine Orientierung & 0,98 & $0,49 * * *$ \\
\hline \multicolumn{3}{|l|}{ Kontrollvariablen } \\
\hline Alter (in Jahren) & $1,39 * * *$ & $1,30 * * *$ \\
\hline Alter ${ }^{2}$ & $0,99 * * *$ & $0,99 * * *$ \\
\hline \multicolumn{3}{|l|}{ Schulischer und beruflicher Ausbildungsgrad (Ref.: Berufsausbildung) } \\
\hline Berufsausbildung + Abitur & $3,16 * * *$ & $3,95 * * *$ \\
\hline Fachhochschulabschluss & $10,00 * * *$ & $8,86 * * *$ \\
\hline Betriebszugehörigkeit 2 Jahre und mehr (Ref.: weniger als 2 Jahre) & $1,55 * * *$ & $1,75 * * *$ \\
\hline Kleinbetriebe (Ref.: Großbetriebe mit 100 und mehr Beschäftigten) & $0,37 * * *$ & $0,68 * * *$ \\
\hline Anteil der Neueinstellungen (in Prozent) & 0,98 & $0,97 * * *$ \\
\hline Betriebsrat (Ref.: kein Betriebsrat vorhanden) & $1,51 * *$ & $2,07 * * *$ \\
\hline $\mathrm{N}$ (Individuen) & 572.004 & 196.327 \\
\hline $\mathrm{N}$ (Betriebe) & 6.616 & 4.267 \\
\hline Log-Likelihood & -306164 & -57769 \\
\hline Wald chi2 (18) & 8793 & 5936 \\
\hline
\end{tabular}

${ }^{* * *} p<0.01, * * p<0.05 ; * p<0.10$.

Anmerkungen: Normalarbeitnehmer (Personen in abhängiger, sozialversicherungspflichtiger Vollzeitbeschäftigung mit mittlerem Ausbildungsgrad).

Anmerkungen: Normalarbeitnehmer (Personen in abhängiger, sozialversicherungspflichtiger Vollzeitbeschättigung mit mittlerem Ausbildungsgrad). Ernährerlohn (Normalarbeitnehmer mit mindestens einem mittlere

bilbranche mit mindestens zweijähriger Betriebszugehörigkeit).
Die Standardfehler sind nach Betrieben geclustert. Andere Wirtschaftsbranchen wurden als binäre Variable in die Modelle mit aufgenommen aber in der Tabelle nicht ausgewiesen.

Die „relativen Chancen“ (engl. „odds ratios“) geben die Chance einer Gruppe auf einen Ernährerlohn im Verhältnis zu einer Referenzgruppe an. Ein Wert unter 1 bedeutet negative, über 1 positive relative Chancen.

oder nicht. Die Chance von Normalarbeitnehmern, in einem frauendominierten Betrieb einen Ernährerlohn zu erzielen, ist wesentlich niedriger als in einem männerdominierten Betrieb. Ähnliches zeigt sich für Beschäftigte in integrierten Betrieben, also dort, wo Frauen und Männer zu gleichen Teilen beschäftigt werden. Für Beschäftigte in Ostdeutschland ergeben sich anders als erwartet keine Unterschiede und dies obwohl frauendominierte Betriebe dort eine weitaus prominentere Stellung einnehmen als in Westdeutschland. Dies mag aus der einerseits sehr unterschiedlichen wirtschaftlichen Struktur in Ostdeutschland, d. h. einem hohen Anteil von Dienstleistungsbeschäftigung mit niedrigerem Lohnniveau, andererseits aus traditionell geringerer Lohn- ungleichheit zwischen den Geschlechtern resultieren.

Einen bedeutenden Einfluss übt die Tarifbindung aus: Die Tatsache, dass in einem Betrieb Löhne auf Basis eines Tarifvertrages gezahlt werden, trägt in beiden Regionen entscheidend zur Chance auf einen Ernährerlohn bei (H3a). Die Form der Tarifbindung, branchenweit oder firmenspezifisch, spielt dabei keine Rolle. Für Ostdeutschland gilt: Dort, wo Beschäftigte in Normalarbeitsverhältnissen in Betrieben ohne Tarifvertrag mit Orientierung am Branchen-Tarifvertrag arbeiten, ist die Chance auf einen Ernährerlohn niedriger als für Beschäftigte in Betrieben mit Branchentarifvertrag. Für diejenigen, die in Betrieben ohne Tarifvertrag und ohne Orientierung an Tarifverträgen beschäftigt sind, liegt sie sogar 
noch darunter. In Westdeutschland ist der Einfluss der Tarifbindung allerdings weniger eindeutig. Dort lassen sich auch Betriebe ohne jegliche Tarifbindung finden, in denen die Chance der dort beschäftigten Normalarbeitnehmer auf Ernährerlöhne ähnlich derer in Betrieben mit Branchentarifbindung ist.

Im Folgenden wird gezeigt, inwieweit der Einfluss der geschlechtsspezifischen Arbeitsmarktsegregation sowie der Tarifbindung auf das Erzielen eines Ernährerlohns bei Frauen und Männern in Normalarbeitsverhältnissen unterschiedlich wirksam ist. Um derartige Interaktionseffekte abzubilden, wird wie folgt vorgegangen: Für jede Ausprägung der Variablen - Branchen, betrieblicher Frauenanteil und Tarifbindung - werden, getrennt für Frauen und Männer in Ost- und Westdeutschland, die vorhergesagten Chancen (Odds), einen Ernährerlohn zu erhalten, ausgewiesen (Tabelle 2). ${ }^{(B)}$ Aus Darstellungsgründen sortieren wir die Chancen dabei nach der Rangfolge ihrer Stärke in Westdeutschland. Zuoberst stehen somit diejenigen Normalarbeitnehmer, welche die größte Chance haben, einen Ernährerlohn zu erwirtschaften. Zwar sortieren sich die Chancen in Ostdeutschland der groben Tendenz nach ähnlich, dennoch werden so auch Unterschiede im Vergleich zu Westdeutschland sichtbar.

Wie sich zeigt, haben vor allem Männer in Westdeutschland in männlich dominierten, aber auch geschlechterintegrierten Branchen und darüber hinaus in tarifgebundenen Betrieben eine hohe Chance, einen Ernährerlohn zu erzielen. Dies gilt für Frauen nur dann, wenn sie in Westdeutschland in den Männerbranchen oder -betrieben beschäftigt sind.

Insgesamt zeigt sich, dass Geschlechterdifferenzen beim Erhalt eines Ernährerlohns unter anderem auf die Positionierung im Beschäftigungssystem anhand der Branchen zurückzuführen sind (Tabelle 2, Modell 1). Es bestätigt sich die Vermutung, dass Frauen in Westdeutschland generell, d. h. unabhängig vom Frauenanteil der Branche, benachteiligt sind $(H 2 b)$. Neben der Geschlechterhierarchie zeigt sich in Westdeutschland aber auch eine Branchenhierarchie: In den kommerziellen Branchen, Industrie wie Dienstleistung, ist die Chance auf einen Ernährerlohn für beide Geschlechter höher als in den öffentlichen, gemeinnützig orientierten und zugleich frauendominierten Dienstleistungsbranchen wie dem Gesundheits- und Sozialwesen. Anders als erwartet, sind die Geschlechterdifferenzen im Banken- und Versicherungswesen am größten. Dies ist vermutlich einem Schließungsprozess gegenüber der Konkurrenz der Frauen geschuldet. In Ostdeutschland trifft der Befund einer generellen Benachteiligung der Frauen für beide Dienstleistungsbranchen, nicht aber für die Metall- und Automobilindustrie zu. Zudem werden auch hier Normalarbeitnehmer im Gesundheits- und Sozialwesen gegenüber den kommerziellen Industrie- und Dienstleistungsbranchen benachteiligt $(\mathrm{H} 2 \mathrm{~b})$.

Darüber hinaus offenbart der Zusammenhang zwischen Geschlecht und betrieblichem Frauenanteil (Tabelle 2, Modell 2) für Westdeutschland Folgendes: Für beide Geschlechter sinkt mit dem Anstieg des Anteils von Frauen im Betrieb die Chance auf einen Ernährerlohn. Insgesamt haben Män- ner in männerdominierten Betrieben höhere Chancen, Frauen in frauendominierten Betrieben hingegen geringe Chancen, einen Ernährerlohn zu erwirtschaften. Auch bestätigt sich, dass es vor allem Frauen sind, die in frauendominierten Betrieben mit Lohneinbußen rechnen müssen. Aber auch in den männerdominierten Betrieben erzielen Frauen seltener einen Ernährerlohn als Männer. Zugleich kann für die vorliegende Stichprobe festgestellt werden, dass Frauen in männerdominierten Betrieben eher von Ernährerlöhnen profitieren als Männer in frauendominierten Betrieben. Für Ostdeutschland gilt: Frauen sind unabhängig vom Anteil von Frauen im Betrieb benachteiligt. Hier trägt somit eher die branchenspezifische Segregation als die betriebliche geschlechtsspezifische Segregation zur Erklärung der ungleichen Verbreitung von Ernährerlöhnen bei.

Was den Zusammenhang von Geschlecht mit Tarifbindung betrifft (Tabelle 2, Modell 3), so zeigt sich in Westdeutschland ein eindeutiger Geschlechtereffekt. Frauen sind in jeder Form von Tarifregime gegenüber den Männern benachteiligt, obgleich sie innerhalb der Gruppe der Frauen von der Tarifbindung profitieren $(H 3 b)$. In Ostdeutschland profitieren Frauen stärker von der Tarifbindung: Anders als in Westdeutschland haben hier Frauen, die in Betrieben mit Tarifvertrag arbeiten, und Männer in Betrieben ohne Tarifvertrag die gleichen Chancen, einen Ernährerlohn zu erzielen.

\section{Fazit}

Nur eine Minderheit der berufsfachlich qualifizierten Normalarbeitnehmer in Deutschland erreicht zwischen 1996 und 2008 überhaupt Ernährerlöhne. Dies sind in erster Linie die westdeutschen Männer - mit weitem Abstand zu den Frauen. Das Lohnniveau in Ostdeutschland ist indes so niedrig, dass Ernährerlöhne selten erreicht werden. Aber auch dort erzielen Frauen seltener einen Ernährerlohn als Männer, wenngleich die geschlechtsspezifische Differenz geringer ist als in Westdeutschland. Der gesamtdeutsch zu beobachtende Prozess der allmählichen Nivellierung dieser Unterschiede findet zulasten der Männerlöhne statt. Dies könnte einerseits ein erster Hinweis auf eine dauerhafte Absenkung von Männerlöhnen sein. Zugleich können andererseits Löhne, die familiale Bedarfe absichern sollen, vor allem von Frauen kaum erreicht werden.

(12) Die Darstellung derTabelle 2 zugrunde liegenden Interaktionsmodelle in Form von Odds Ratios (vgl. Tabelle 1) ist problematisch, da es sich bei den Koeffizienten um bedingte Effekte handelt. Um verschiedene Gruppen (etwa Frauen in frauendominierten Branchen mit Männern in frauendominierten Branchen) miteinander vergleichen zu können, lösen wir die Logit-Gleichung deshalb auf und erhalten so für jede Gruppe die vorhergesagten Odds, d. h. die Chance dieser Gruppe, einen Ernährerlohn zu erhalten. 


\section{Determinanten des Ernährerlohns bei Normalarbeitnehmern: Interaktion von Geschlecht mit Segregation und Tarifbindung 2008}

Logit-Regression, vorhergesagte Chancen

\begin{tabular}{|c|c|c|}
\hline & Westdeutschland & Ostdeutschland \\
\hline \multicolumn{3}{|l|}{ Branchen (Modell 1) } \\
\hline Männer x Banken und Versicherungen & 3,73 & 1,13 \\
\hline Männer x Metall und Automobilbranche & 3,29 & 0,24 \\
\hline Frauen $\times$ Metall und Automobilbranche & 2,03 & 0,28 \\
\hline Frauen x Banken und Versicherungen & 0,67 & 0,34 \\
\hline Männer x Gesundheits- und Sozialwesen & 0,23 & 0,07 \\
\hline Frauen x Gesundheits- und Sozialwesen & 0,05 & 0,02 \\
\hline \multicolumn{3}{|l|}{ Betrieblicher Frauenanteil (Modell 2) } \\
\hline Männer x Männerdominierter Betrieb & 2,50 & 0,20 \\
\hline Männer x Integrierter Betrieb & 1,93 & 0,33 \\
\hline Frauen x Männerdominierter Betrieb & 1,10 & 0,18 \\
\hline Männer x Frauendominierter Betrieb & 0,49 & 0,25 \\
\hline Frauen x Integrierter Betrieb & 0,40 & 0,13 \\
\hline Frauen $\times$ Frauendominierter Betrieb & 0,07 & 0,07 \\
\hline \multicolumn{3}{|l|}{ Tarifbindung (Modell 3) } \\
\hline Männer x Branchentarifvertrag & 2,50 & 0,32 \\
\hline Männer x Firmentarifvertrag & 2,30 & 0,25 \\
\hline Männer x Kein Tarifvertrag, keine Orientierung am Branchentarifvertrag & 1,40 & 0,08 \\
\hline Männer x Kein Tarifvertrag, Orientierung an Branchentarifvertrag & 0,96 & 0,10 \\
\hline Frauen x Firmentarifvertrag & 0,62 & 0,11 \\
\hline Frauen $\times$ Branchentarifvertrag & 0,48 & 0,12 \\
\hline Frauen x Kein Tarifvertrag, keine Orientierung am Branchentarifvertrag & 0,32 & 0,03 \\
\hline Frauen x Kein Tarifvertrag, Orientierung an Branchentarifvertrag & 0,15 & 0,05 \\
\hline
\end{tabular}

Anmerkungen: Normalarbeitnehmer: Personen in abhängiger, sozialversicherungspflichtiger Vollzeitbeschäftigung mit mittlerem Ausbildungsgrad.

Ernährerlohn: Normalarbeitnehmer mit mindestens einem mittleren Tages-Brutto-Reallohn eines männlichen Normalarbeitnehmers in der Metall- oder Automobilbranche mit mindestens zweijähriger Betriebszugehörigkeit.

Hinweis: Es wurden je für West- und Ostdeutschland drei Modelle mit den jeweiligen Interaktionsvariablen (Geschlecht und Branche, Geschlecht und Frauenanteil im Betrieb, Geschlecht und Tarifbindung) gerechnet. Die Standardfehler sind nach Betrieben geclustert. Andere Wirtschaftsbranchen wurden als binäre Variable in die Modelle mit aufgenommen, aber in der Tabelle nicht ausgewiesen. Die Kontrollvariablen (siehe Tabelle 1) wurden in die Modelle aufgenommen, werden jedoch nicht ausgewiesen.

Bei den „vorhergesagten Chancen“ (engl. „odd“) handelt es sich um die Wahrscheinlichkeit, einen Ernährerlohn zu erhalten, im Verhältnis zur Wahrscheinlichkeit, ihn nicht zu erhalten. Werte über 1 stellen positive, unter 1 negative Chancen dar. Die Chancen der jeweiligen Modelle werden in der Reihenfolge ihrer Stärke am Maßstab Westdeutschlands dargestellt.

Quelle: LIAB Querschnittmodell (Version 2), 2008, ungewichtete Ergebnisse, Berechnungen der Autoren.

In Ost- wie Westdeutschland haben Beschäftigte in den hier betrachteten kommerziellen Industriebranchen mit vorwiegender Männerbeschäftigung weitaus bessere Aussichten auf Ernährerlöhne als in den durch öffentliche bzw. gemeinnützige Arbeitgeber gekennzeichneten sozialen Dienstleistungen mit vorwiegender Frauenbeschäftigung wie dem Gesundheits- und Sozialwesen (vgl. Bispinck/WSITarifarchiv in diesem Heft). Doch auch und gerade in den geschlechterintegrierten kommerziellen Dienstleistungsbranchen, den Banken und Versicherungen, werden häufiger Ernährerlöhne als in den sozialen Dienstleistungsbranchen erreicht. Die Geschlechterunterschiede sind dort - vor allem in Westdeutschland - besonders groß. Unabhängig von den Branchen wirkt sich ein hoher Frauenanteil im Betrieb, gerade in Westdeutschland und insbesondere für Frauen, ne- gativ auf die Möglichkeit aus, einen Ernährerlohn zu erhalten. Herauszustellen ist darüber hinaus die positive Wirkung der institutionellen Interessenvertretung: Beschäftigte in Betrieben mit Tarifbindung erzielen unabhängig von der Form des Tarifvertrages (branchenweit oder firmenspezifisch) öfter einen Ernährerlohn - vor allem in Regionen mit geringer Tarifabdeckung wie Ostdeutschland. Zugleich jedoch fällt diese positive Wirkung fast ausschließlich zugunsten der Männer aus. Somit ergeben sich je nach Branche, Frauenanteil im Betrieb und Tarifbindung ganz unterschiedliche Defizite in Bezug auf höhere Löhne, d. h. Löhne, die neben der individuellen Existenzsicherung auch Kosten zur Bedarfsdeckung einer Familie beinhalten sollen.

Insgesamt lassen die Ergebnisse mit Blick auf die Löhne auf eine Kontinuität der regional unterschiedlich tra- 
dierten Ernährermodelle im Erwerbssystem schließen. In Westdeutschland führen die strukturelle Positionierung der Geschlechter in Branchen und Betrieben sowie die für Frauen und Männer unterschiedliche institutionelle Wirkung der Interessenvertretung von Arbeitnehmern zu großen geschlechtsspezifischen Lohndifferenzen. Selbst vollzeiterwerbstätige, berufsfachlich qualifizierte Frauen können in bestimmten Branchen, insbesondere dem expandierenden Sozial- und Gesundheitsbereich, für den zum Teil schon heute Fachkräftemangel konstatiert wird, keinen Ernährerlohn erzielen. Dies lässt unter gegenwärtigen Bedingungen die Realisierungschancen von eher egalitär ausgerichteten und individualisierten Leitbildern, die auf eigenständige Existenzsicherung durch Erwerbsarbeit auch für Frauen zielen, fraglich erscheinen. In Ostdeutschland zeigt sich ein ähnliches Bild auf geringerem Niveau, wobei die Frauen stärker von der Tarifbindung profitieren und die Auswirkungen der geschlechtsspezifischen Arbeitsmarktsegregation weitaus geringer sind. Doch auch hier bestehen geschlechtsspezifische Lohndifferenzen mit Blick auf den Ernährerlohn. Dies erscheint nicht zuletzt angesichts des ohnehin geringen Lohnniveaus bedenklich.

Für die tarifpolitischen Akteure - Arbeitgebervertreter wie Gewerkschaften gleichermaßen - ergibt sich die Herausforderung, auf den Abbau geschlechtsspezifischer Lohnungleichheit hinzuwirken. Dies würde nicht nur dem lohnpolitischen Prinzip gleichen Lohns für gleichwertige Arbeit entsprechen, sondern könnte auch dazu beitragen, das lange tradierte, gleichwohl überkommene Leitbild des männlichen Familienernährers zugunsten einer stärker egalitären Arbeitsteilung zwischen Männern und Frauen in Erwerbsarbeit und Familie zu überwinden und stattdessen eher modernen Familienkonstellationen, wie bspw. alleinerziehenden Frauen oder Familienernährerinnen, Rechnung zu tragen. Eine besondere tarifpolitische Herausforderung ergibt sich für die meist öffentlichen oder gemeinnützigen Arbeitgeber und Gewerkschaften im Bereich sozialer Dienstleistungen, wo fachlich qualifizierte Arbeitskräfte in Vollzeitarbeit bisher kaum Löhne erzielen können, die eine eigenständige und familienorientierte Existenzsicherung erlauben. Die Einführung eines Mindestlohns in der Pflegebranche sowie das Urteil des Bundesarbeitsgerichts zum Streikrecht in kirchlichen Einrichtungen können hier nur erste Schritte sein. Die offensichtlich verbreitete geschlechtsspezifische Lohnungleichheit auch jenseits von Mindestlöhnen verweist schließlich auch auf weiteren genuin gleichstellungspolitischen Handlungsbedarf - etwa in Form der Einführung betrieblicher Gleichstellungsmaßnahmen. Dabei ist freilich eine bloße Umverteilung zwischen den Geschlechtern zugunsten der Unternehmen und zulasten der Familien zu vermeiden.

\section{LITERATUR}

Alda, H. (2005): Betriebe und Beschäftigte in den Linked-Employer-EmployeeDaten: LIAB des Instituts für Arbeitsmarkt- und Berufsforschung, FDZ-Datenreport (01), Nürnberg

Andreß, H.-J./Seeck, T. (2007): Ist das Normalarbeitsverhältnis noch armutsvermeidend? Erwerbstätigkeit in Zeiten deregulierter Arbeitsmärkte und des Umbaus sozialer Sicherungssysteme, in: Kölner Zeitschrift für Soziologie und Sozialpsychologie 59 (3), S. 459-492

Antonczyk, D./Fitzenberger, B./Sommerfeld, K. (2011): Anstieg der Lohnungleichheit, Rückgang der Tarifbindung und Polarisierung, in: Zeitschrift für Arbeitsmarktforschung 44 (1/2), S. 15-27

Beck-Gernsheim, E. (1981): Der geschlechtsspezifische Arbeitsmarkt: zur Ideologie und Realität von Frauenberufen, Frankfurt a. M. et al.

Beckord, J. (1977): Lohnführerschaft und kollektive Lohnverhandlung, Frankfurt a. M.
Bonin, H. (2005): Tarifpolitik und Entgeltflexibilität in Ostdeutschland, IZA Discussion Paper (1871), Bonn

Brenke, K./Grabka, M. M. (2011): Schwache Lohnentwicklung im letzten Jahrzehnt, in: DIW Wochenbericht 84 (45), S. 3-15

Busch, U./Land, R. (2012): Ostdeutschland. Vom staatssozialistischen Fordismus in die Entwicklungsfalle einerTransferökonomie, in: Forschungsverbund Sozioökonomische Berichterstattung (Hrsg.): Berichterstattung zur sozioökonomischen Entwicklung in Deutschland. Teilhabe im Umbruch. Zweiter Bericht Wiesbaden

Dietz, M./Walwei, U. (2006): Beschäftigungswirkungen des Wandels der Erwerbsformen, in: WSI-Mitteilungen 59 (5), S. 278-286, http://www.boeckler. de/wsimit_2006_05_dietz.pdf

Dustmann, C./Ludsteck, J./Schönberg, U. (2009): Revisiting the German Wage Structure, in: The Quarterly Journal of Economics 124 (2), S. 843-881

Falk, S. (2005): Geschlechtsspezifische Ungleichheit im Erwerbsverlauf - Analysen für den deutschen Arbeitsmarkt, Wiesbaden

Finke, C. (2011): Verdienstunterschiede zwischen Männern und Frauen 2006, Wiesbaden

Gartner, H./Rässler, S. (2005): Analyzing the Changing Gender Wage Gap based on Multiply Imputed Right Censored Wages, IAB-Discussion Paper 5/2005, Nürnberg

Hauser, R./Wagner, G. (1996): Die Einkommensverteilung in Ostdeutschland Darstellung, Vergleich und Determinanten für die Jahre 1990 bis 1994, in: Hau ser, R./Lampert, H./Ribhegge, H./Wagner, G./Zerche, J. (Hrsg.): Sozialpolitik im vereinten Deutschland. Bd. 3. Familienpolitik, Lohnpolitik und Verteilung, Berlin, S. 79-127

Heinze, A. (2009): Earnings of men and women in firms with a female dominated workforce. What drives the impact of sex segregation on wages?, ZEW Discussion paper 09-012, Mannheim

Hinrichs, K. (1996): Das Normalarbeitsverhältnis und der männliche Familienernährer als Leitbilder der Sozialpolitik. Sicherungsprobleme im Wandel, in: Sozialer Fortschritt 45 (4), S.102-107

Hunt, J. (2002): The Transition in East Germany: When is a Ten-Point Fall in the Gender Wage Gap Bad News?, in: Journal of Labor Economics 20 (1),

S. $148-169$

Jacobebbinghaus, P./Seth, S. (2010): Linked-Employer-Employee-Daten des IAB: LIAB-Querschnittmodell 2 1993-2008 (LIAB QM2 9308), FDZ-Datenreport (05), Nürnberg

Klenner C./Menke, K./Pfahl, S. (2012): Flexible Familienernährerinnen, Berlin/ Opladen

Krueger, A. B./Pischke, J.-S. (1995): A Comparative Analysis of East and West German Labor Markets: Before and After Unification, in: Freeman, R. B./Katz, L. F. (Hrsg.): Differences and Changes in Wage Structures, Chicago,

S. $405-446$

Lappe, L. (1981): Die Arbeitssituation erwerbstätiger Frauen, Frankfurt a. M./ New York

Lewis, J./Ostner, I. (1994): Gender and the Evolution of European Social Policies, ZeS-Arbeitspapier 4/94, Bremen

Lohmann, H./Andreß, H.-J. (2011): Autonomie oder Armut? Zur Sicherung gleicher Chancen materieller Wohlfahrt durch Erwerbsarbeit, in: WSI-Mitteilungen 64 (4), S.178-187, http://www.boeckler.de/wsimit_2011_04_Andress.pdf

Long, J. S./Freese, J. (2006): Regression models for categorical dependent variables using Stata, College Station, Texas

Mayer-Ahuja, N./Bartelheimer, P./Kädtler, J. (2012): Teilhabe im Umbruch - Zur sozioökonomischen Entwicklung in Deutschland, in: Forschungsverbund Sozioökonomische Berichterstattung (Hrsg.): Berichterstattung zur sozio-ökonomischen Entwicklung in Deutschland. Teilhabe im Umbruch. Zweiter Bericht, Wiesbaden, S. 15-39

Mückenberger, U. (1989): Der Wandel des Normalarbeitsverhältnisses unter Bedingungen einer „Krise der Normalität”, in: Gewerkschaftliche Monatshefte 40 (4), S. $211-223$

Peuckert, R. (2008): Familienformen im sozialen Wandel, Wiesbaden

Roloff, J. (1991): Probleme und Ursachen der Einkommensunterschiede zwischen männlichen und weiblichen Erwerbstätigen in der ehemaligen DDR, in: Zeitschrift für Bevölkerungswissenschaft 17 (1), S. 135-141

Roos, M. W. M. (2006): Earnings Disparities in Unified Germany: Nominal versus Real, in: Jahrbuch für Regionalwissenschaft 26 (2), S.171-189

Schlegel, U. (1995): Ostdeutsche Frauen in neuen gesellschaftlichen Strukturen, in: Sydow, H./Schlegel, U./Helmke, A. (Hrsg.): Chancen und Risiken im Lebenslauf, Opladen, S. 111-128 
Statistisches Bundesamt (2012): Wirtschaftsrechnungen. Laufende Wirtschaftsrechnungen Einnahmen und Ausgaben privater Haushalte 2010, Wiesbaden

Streeck, W. (1995): German Capitalism: Does It Exist? Can It Survive?, MPIfG Discussion Paper 95/5, Köln

Strengmann-Kuhn, W. (2003): Armut trotz Erwerbstätigkeit. Analysen und sozialpolitische Konsequenzen, Frankfurt a. M.

Szydlik, M. (1993): Arbeitseinkommen und Arbeitsstrukturen. Eine Analyse für die Bundesrepublik Deutschland und die Deutsche Demokratische Republik, Berlin

Trappe, H./Rosenfeld, R. A. (2001): Geschlechtsspezifische Segregation in der DDR und der BRD. Im Verlauf der Zeit und im Lebensverlauf. Geschlechtersoziologie, in: Kölner Zeitschrift für Soziologie und Sozialpsychologie, Sonderheft 41, S. $152-181$

Trappe, H./Sørensen, A. (2006): Economic Relations between Women and Their Partners: An East and West German Comparison after Reunification, in: Feminist Economics 12 (4), S. 643-665

Winkler, G. (1990): Frauenreport '90, Berlin
AUTOREN

TIM SCHRÖDER, Mag. Soz., Wissenschaftlicher Mitarbeiter am Zentrum für Sozialpolitik, Universität Bremen, im Projekt der Hans-Böckler-Stiftung „Was kommt nach dem Familienlohn?" (Projektnr. 2010-375-4). Arbeitsschwerpunkte: Lebensverlaufsforschung, Lohn und Beschäftigung, regionale Mobilität.

t.schroeder@zes.uni-bremen.de

ANDREA SCHÄFER, Mag. Soz., Wissenschaftliche Mitarbeiterin am Zentrum für Sozialpolitik, Universität Bremen. Arbeitsschwerpunkte: Arbeitsmarkt- und (quantitative) Geschlechterforschung.

@a.schaefer@zes.uni-bremen.de 